\title{
A REDUCTION OF THE SCHOENFLIES EXTENSION PROBLEM
}

BY MARSTON MORSE

Communicated by Edwin Moise, December 28, 1959

1. In his study of the Schoenflies extension problem Mazur, [1], includes the hypothesis that the given mapping $\phi$ be "semilinear" in a neighborhood of some point in the shell domain of $\phi$. In Mazur's paper the problem of removing this hypothesis is unsolved. We shall show how to remove this hypothesis, leaving the "shell hypothesis." In the process of doing this we establish two fundamental lemmas concerning the equivalence of extension problems.

Let $E$ and $\varepsilon$ be euclidean $n$-spaces, $n>1$, with rectangular coordinates $(x)$ and $(y)$ respectively. Let $(x)$ and $(y)$ be represented by vectors $x$ and $y$ with components $\left(x_{1}, \cdots, x_{n}\right)$ and $\left(y_{1}, \cdots, y_{n}\right)$ respectively. Let $\|x\|$ be the distance of the point $x$ from the origin. Let $I$ be the mapping of $E$ onto $\varepsilon$ under which $x=y$. Let $S_{n-1}$ be an $(n-1)$-sphere of unit radius in $E$ with center at the origin. Let J $S_{n-1}$ be the interior of $S_{n-1}$. With $0<a<1$, introduce the $n$-shell

$$
\sigma_{a}=\{x \mid 1-a<\|x\|<1+a\} .
$$

As applied to a mapping of an open subset of a euclidean space or a differentiable manifold into a similar space, a $C^{m}$-diffeomorphism shall have its usual meaning when $m>0$, but when $m=0$ shall be merely a homeomorphism.

In this sense let

$$
\phi: \sigma_{a} \rightarrow \varepsilon
$$

be a $C^{m}$-diffeomorphism of $\sigma_{a}$ onto a subset of $\varepsilon$. The set $\phi\left(\sigma_{a}\right)$ is open in $\varepsilon$ and affords a neighborhood of the manifold $\phi\left(S_{n-1}\right)=\Re_{n-1}$. Let ${ }^{J} \mathfrak{T T}_{n-1}$ be the interior of $\mathfrak{T T}_{n-1}$. We assume that $\phi$ carries points of $\sigma_{a}$ which are interior to $S_{n-1}$ into points which are interior to $\mathfrak{T}_{n-1}$. We term these conditions on $\phi$ the shell hypothesis.

THEOREM 1. (i) If $N$ is a sufficiently small neighborhood of $S_{n-1}$ there exists an extension $\Lambda_{\phi}$ of $\phi \mid N$ which is a homeomorphism of

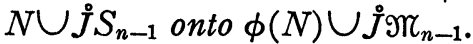

(ii) If $m>0$ and if $Z$ is an arbitrary point interior to $S_{n-1}$, then $\Lambda_{\phi}$ and $N$ may be chosen so that $\Lambda_{\phi}$ is, in addition, a $C^{m}$-diffeomorphism of $N \cup \dot{J}\left(S_{n-1}\right)-Z$ into $\varepsilon$.

In the differentiable case $(m>0)$, the theorem has been proved by the author in [2]. In the topological case $(m=0)$ we shall give a 
proof of (i) by transforming the problem into an equivalent problem solvable by the methods of Mazur in [1], or by the subsequent methods of the author in [2].

Given the $C^{m}$-diffeomorphism $\phi$ of Theorem 1, we term a mapping $\Lambda_{\phi}$ which satisfies Theorem 1 a solution of the Schoenflies extension problem $\left[\phi, \sigma_{a}\right]$. Let $f$ be a $C^{m}$-diffeomorphism of the set

$$
\phi\left(\sigma_{a}\right) \cup \dot{J}_{\phi}\left(S_{n-1}\right)=\Omega
$$

into $\varepsilon$. It must be remembered that when $m=0$, a $C^{m}$-diffeomorphism is merely a homeomorphism.

Lemma 1. (i) If $\phi$ is $a C^{m}$-diffeomorphism of $\sigma_{a}$ into \& satisfying the shell hypothesis, $m \geqq 0$, then fo is a $C^{m}$-diffeomorphism of $\sigma_{a}$ into $\varepsilon$ satisfying the shell hypothesis.

(ii) A necessary and sufficient condition that there exist a solution $\Lambda_{\phi}$ of the problem $\left[\phi, \sigma_{a}\right]$ is that there exist a solution $\Lambda_{f \phi}$ of the problem $\left[f \phi, \sigma_{a}\right]$.

Proof of (i). It is clear that $f \phi$ is a $C^{m}$-diffeomorphism of $\sigma_{a}$ into $\varepsilon$. Set $f\left(\phi\left(S_{n-1}\right)\right)=\mathfrak{L}_{n-1}$. We must show that $f \phi$ carries a point $\mathrm{x}$ of $\sigma_{a}$ interior to $S_{n-1}$ into a point interior to $\mathfrak{L}_{n-1}$.

This will follow once we have proved the relation

$$
f\left({\left.\stackrel{\circ}{J} \mathfrak{T}_{n-1}\right)}={\stackrel{\circ}{J} \mathfrak{L}_{n-1}} .\right.
$$

Now ${\stackrel{\circ}{J} T_{n-1}}_{\text {is }}$ the unique, bounded, maximal connected subset of $\varepsilon$ which does not meet $\mathfrak{T}_{n-1}$. Set $f\left(\mathfrak{J}_{\mathfrak{H}} \mathfrak{H}_{n-1}\right)=W$. The image set $W$ is bounded and connected. Making use of the fact that $f$ is locally a $C^{m_{-}}$ diffeomorphism of open subsets of $\varepsilon$ onto open subsets of $\varepsilon$, one can verify the definition of $f^{-1}$ along all paths in $\varepsilon$ that start with points of $W$ and do not meet $\mathfrak{L}_{n-1}$. Since the image under $f^{-1}$ remains in $\mathfrak{J}_{\mathfrak{N}}{ }_{n-1}$ these paths are in $W$. Hence $W$ is a maximal, connected subset of $\varepsilon$ that does not meet $\mathfrak{L}_{n-1}$. Since $W$ is bounded $W=J^{\circ} \mathfrak{L}_{n-1}$.

Thus (4) holds. A point $\mathrm{x}$ of $\sigma_{a}$ interior to $S_{n-1}$ has by hypothesis an image $\phi(x)$ interior to $\mathfrak{T}_{n-1}$, and by $(4) f(\phi(x))$ is interior to $\mathfrak{L}_{n-1}$. This establishes (i).

Proof of (ii). Suppose that $\Lambda_{\phi}$ is a solution of problem $\left[\phi, \sigma_{a}\right]$. I say that $f \Lambda_{\phi}$ is a solution of problem $\left[f \phi, \sigma_{a}\right]$. For $f \Lambda_{\phi}$ is an extension of $(f \phi) \mid N$ since $f\left(\Lambda_{\phi}(\mathbf{x})\right)=f(\phi(x))=(f \phi)(\mathbf{x}), \quad[\mathrm{x} \in N]$. Moreover $f \Lambda_{\phi}$ is a homeomorphism of $N \cup \grave{J}\left(S_{n-1}\right)$ onto

$$
\left(f \Lambda_{\phi}\right)\left(N \cup \mathfrak{J}_{n-1}\right)=f(\phi(N)) \cup f\left(J_{\mathfrak{T}} \boldsymbol{T}_{n-1}\right)=(f \phi)(N) \cup \mathfrak{J}_{\mathscr{L}_{n-1}}
$$

in accord with (4). Moreover, if $m>0, f \Lambda_{\phi}$ is a $C^{m}$-diffeomorphism of $N \cup \stackrel{\circ}{J} S_{n-1}-Z$ into $\varepsilon$. 


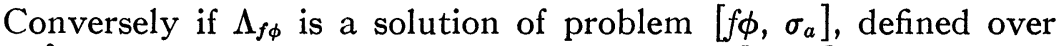
$N \cup \stackrel{J}{J}\left(S_{n-1}\right)$, then $f^{-1} \Lambda_{f \phi}$ is a solution of problem $\left[\phi, \sigma_{a}\right]$, defined over $N \cup \stackrel{j}{J} S_{n-1}$. One makes use of the relation, derived from (4),

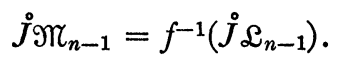

The Нyротнesis A. We say that the given mapping $\phi$ satisfies hypothesis A if $\phi$ reduces to $I$ in some neighborhood $X$, relative to $\sigma_{a}$, of some point $Q$ of $S_{n-1}$.

Lemma 2. Corresponding to a given problem $\left[\phi, \sigma_{a}\right]$ of index $m \geqq 0$, for suitable definition of a $C^{m}$-diffeomorphism $f$ of the set $\Omega$ into $\&$ the $C^{m}$-diffeomorphism fo of $\sigma_{a}$ into \& satisfies Hypothesis A.

Proof of Lemma 2. Let $Q$ be an arbitrary fixed point of $S_{n-1}$. For the purposes of this proof suppose that $\phi(Q)$ is the origin in $\varepsilon$. Let $\mathfrak{F}_{\rho}$ be an open $n$-ball in $\varepsilon$ with center at the origin and radius $\rho$. In particular let $\mathfrak{H}_{2 b}$ have a radius so small that $\mathfrak{H C}_{2 b} \subset \phi\left(\sigma_{a}\right)$. Set

$$
\phi^{-1}\left(\mathfrak{F C}_{b}\right)=X_{b}, \quad \phi^{-1}\left(\mathfrak{H C}_{2 b}\right)=X_{2 b}
$$

and note that $X_{2 b} \subset \sigma_{a}$. Let $t \rightarrow \lambda(t)$ be a $C^{\infty}$-diffeomorphism of the open interval $(0,2 b)$ onto the positive $t$-axis such that $\lambda(t)=t$ for $t \in(0, b)$. Such a mapping exists.

Let $\mu$ be the $C^{\infty}$-diffeomorphism of $\mathfrak{F C}_{2 b}$ onto $\varepsilon$ such that the point $y \in \mathcal{K}_{2 b}$ has the image $\lambda(\|y\|) y /\|y\|$ when $\|y\| \neq 0$ and the image $O$ when $y=O$. Set

$$
\psi(x)=\mu(\phi(x)) \quad\left(x \in X_{2 b}\right),
$$

and note that $\psi(x)=\phi(x)$ for $x \in X_{b}$ and that $\psi\left(X_{2 b}\right)=\varepsilon$. We can accordingly define a $C^{m}$-diffeomorphism $f$ over $\Omega$ by setting

$$
f(y)=I\left(\psi^{-1}(y)\right) \quad(y \in \Omega) .
$$

We have

$$
f(\phi(x))=I(x) \quad\left(x \in X_{b}\right) .
$$

Thus $f \phi$ satisfies Hypothesis A.

Theorem 1 (i) follows from the two lemmas taking account of the known fact that the problem $\left[f \phi, \sigma_{a}\right]$ admits a solution even when $m=0$, since $f \phi$ satisfies, not only the shell hypothesis, but also hypothesis A.

\section{REFERENCES}

1. Barry Mazur, On embeddings of spheres, Bull. Amer. Math. Soc. vol. 65 (1959) pp. 59-65.

2. Marston Morse, Differentiable mappings in the Schoenflies theorem. Compositio Math. vol. 14 (1959) pp. 83-151.

INSTITUTE FOR ADVANCED STUdy 